\title{
Das Lorscher Arzneibuch: von antik-heidnischer Medizin über christliche Heilkunst bis zum UNESCO-Weltdokumentenerbe
}

\author{
Christiane Högermann
}

\begin{abstract}
The medicinal book of Lorsch originates from the early middle age. It is a handwriting dating from time of CHARLES THE Great. This unique book was written in the Abbey of Lorsch. Today it is stored in the library of Bamberg. The book contains abut 500 recipes, some of them were new. It has become UNESCO documentary heritage and the rest of the Abbey of Lorsch is UNESCO world culture heritage.
\end{abstract}

\section{Zusammenfassung}

Das Lorscher Arzneibuch stammt aus dem frühen Mittelalter und ist eine Handschrift aus der Zeit von KarL Dem Grossen. Das einzigartige Werk entstand im Kloster Lorsch, liegt heute in der Bamberger Staatsbibliothek. Das Buch enthält rund 500 verschiedene Rezepturen, für die damalige Zeit auch ganz neue. Aufgrund seiner Einzigartigkeit wurde es in die Liste des UNESCO-Weltdokumentenerbes aufgenommen, so wie auch die Reste der Benediktinerabtei in Lorsch ein UNESCO-Weltkulturerbe darstellen.

\section{Altes Wissen über die Heilkunde}

Am 18. Juni 2013 hat das Lorscher Arzneibuch als ältestes Dokument der Medizingeschichte des abendländischen Mittelalters den Status eines Weltdokumentenerbes verliehen bekommen. Vor etwa 1000 Jahren hatte Kaiser HeInRICH II. dafür gesorgt, dass es nach Bamberg kam.
Dort befindet es sich heute im Besitz der Bamberger Staatsbibliothek. Es handelt sich bei diesem Monumentalwerk um eine Handschrift, deren umfangreiche tradierten heilkundlichen Inhalte faszinieren. Auf (natur-)wissenschaftlicher Ebene hat das Buch Geschichte geschrieben. Neben heute noch anwendbarem Wissen aus der Phytopharmakologie dokumentiert es eindrücklich die Entwicklung der Fachrich-

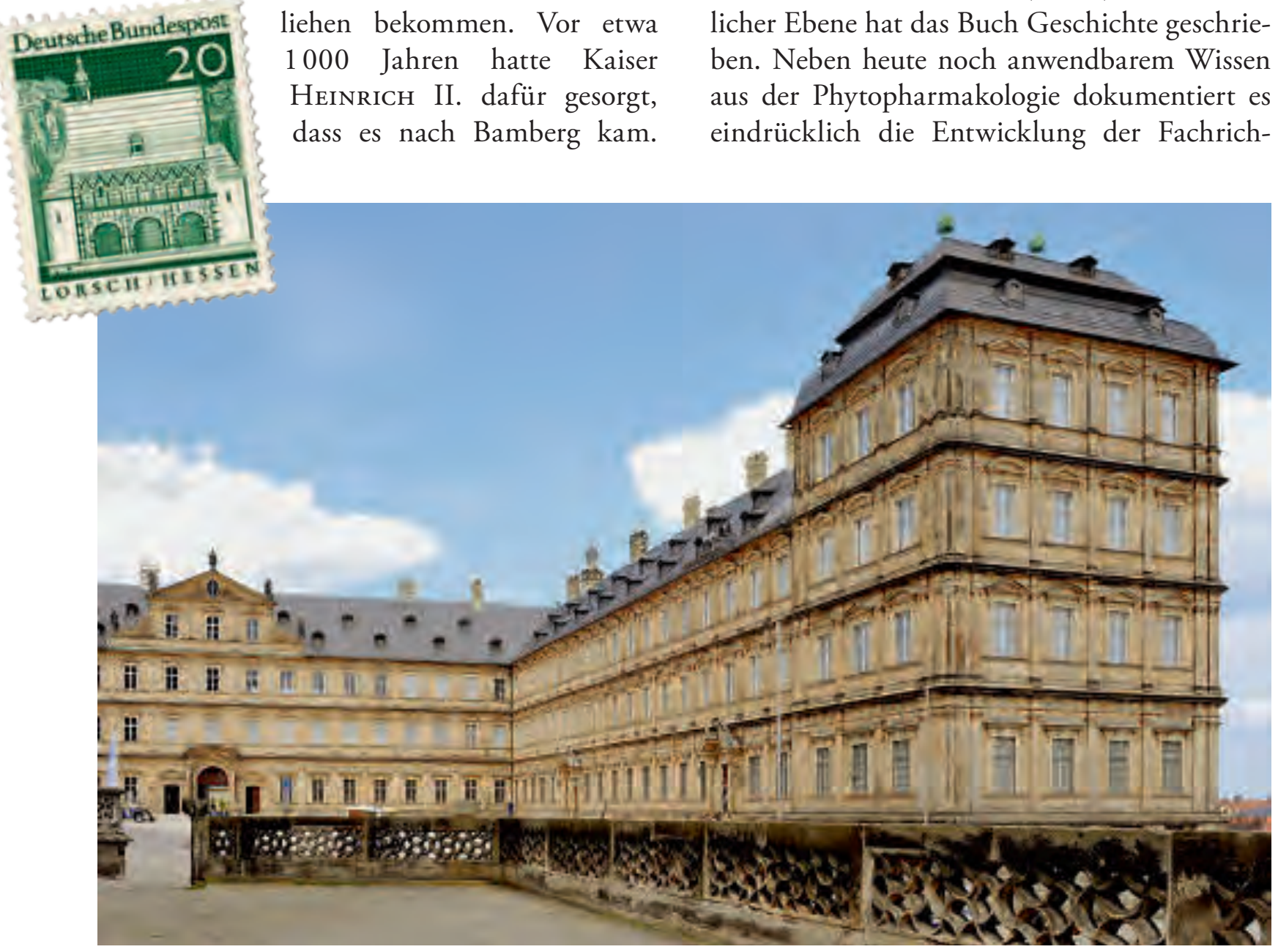


tung. Die Aufzeichnungen stammen aus der südhessischen Benediktinerabtei Lorsch, ihrerseits UNESCO-Weltkulturerbe seit 1991.

\section{Krankheiten, Pflege und Heilmethoden}

Mit dem klösterlichen Entstehungsort ist auch seine theologisch-philosophische Tragweite eng verbunden. In der Antike galt die Behandlung und Heilung von Kranken durch Heilkundige als unerlaubter Eingriff in die Allmacht Gottes. Unter Karl DEM Grossen wandelte sich diese Weltanschauung; der christliche Glaube verpflichtete zur Nächstenliebe und damit auch zum Heilen Kranker. Aber nicht nur der frühmittelalterliche Glaube von Krankheiten als Strafe Gottes bis hin zur Erbsünde wurde unter KarL einer Revision unterzogen, das Werk vermittelt auch ein zeitgenössisches Bild zur Situation der Kranken, ihrer Pflege und Heilung durch Mönchsärzte sowie der Apotheker in karolingischer Zeit.

\section{Inhalte des Werkes}

Das (Kunst-)Werk, konzipiert als Lehr- und Nachschlagewerk (u.a, Geschichte, Anatomie, Drogen-Ersatzliste, Maßeinheiten, Glossar) umfasst als Hauptteil auf ca. 150 Seiten knapp 500 handgeschriebene Rezepturen sowie auf die Heilkunst bezogene Schriften, alles wie damals üblich in lateinischer Sprache verfasst. Einige der noch heute praktizierten Medikationen hatten damals innovativen Charakter, so etwa der

Abb. 1 (Seite 38 oben): Karolingische Torhalle des ehemaligen Klosters Lorsch auf einer 20-PfennigBriefmarke, Ausgabejahr 1966.

Abb. 2 (Seite 38 unten): Die barocke Neue Residenz in Bamberg beherbergt in ihrem Ostflügel die Staatsbibliothek Bamberg, zu deren Bestand das Lorscher Arzneibuch gehört. In diesem Teil der Residenz war früher die fürstbischöfliche Verwaltung angesiedelt.

Abb. 3 (oben): Das Lorscher Arzneibuch in der Staatsbibliothek Bamberg, ein mittelalterlicher Pergamentband.

Abb. 4 (Mitte): Doppelseite im aufgeschlagenen Arzneibuch.

Abb. 5 (unten): Ein im Pergament vorhandenes Loch, das umschrieben wurde.
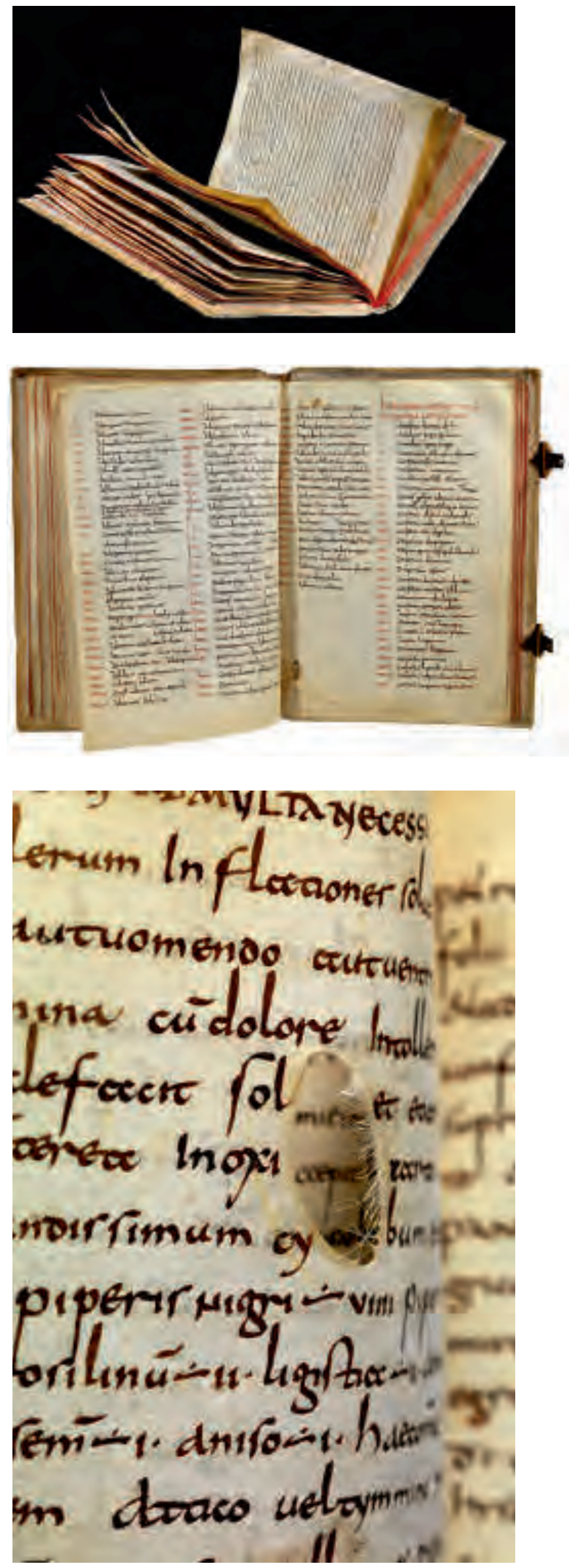


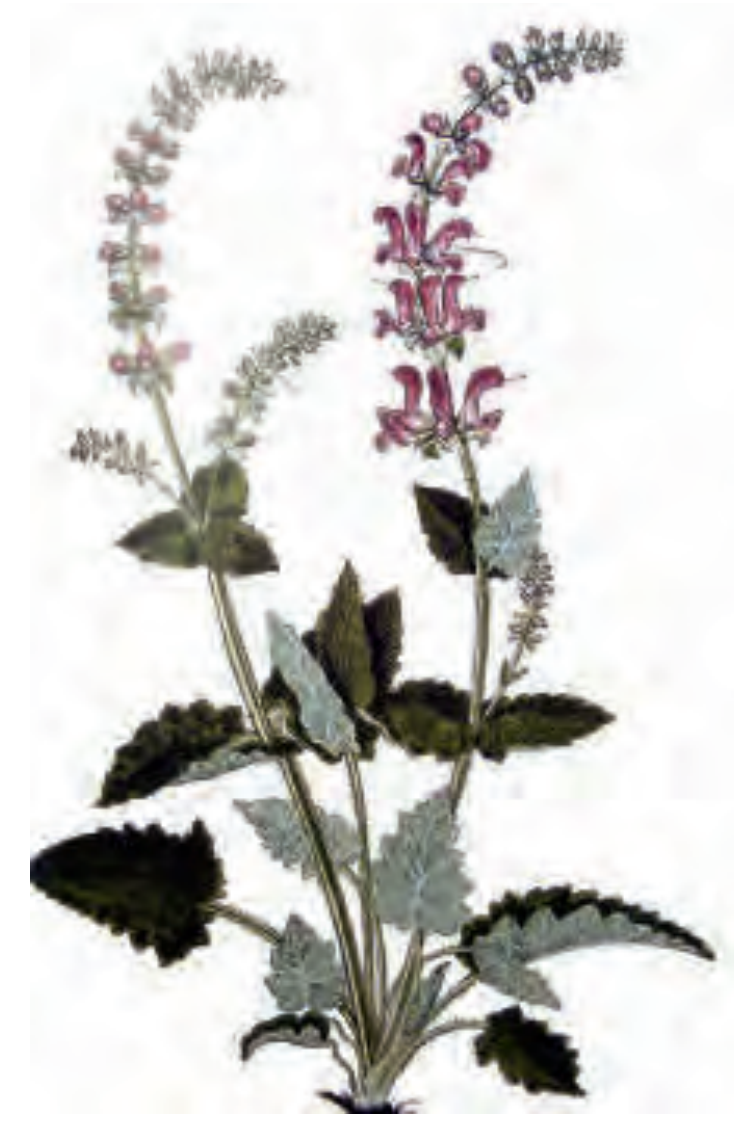

Einsatz von Johanniskraut zur Behandlung in der Psychiatrie und die Verwendung von Herzglykosiden zur Kreislaufstabilisierung. Erwähnenswert ist zudem die in Versform verfasste Anleitung zum Verhältnis von Arzt und Patient. Außerdem wird die Forderung thematisiert, unabhängig vom Reichtum allen Menschen eine medizinische Behandlung zu gewähren.

Im Schlussteil widmet der griechische Arzt Anthimus eine Abhandlung der gesunden Ernährung. Hier zeigt sich, dass dieses Werk auch heute noch seinen Stellenwert in der modernen sowie alternativen Heilkunde besitzt.

Der Text des Arzneibuches zeichnet sich durch die besondere und besonders sorgfältige Umsetzung der karolingischen MinuskelSchriftart aus. Für die karolingische Form der Minuskel, deren Entstehung bis in das 8. Jh. zurückgeht, ist die Einfachheit und Klarheit des Schriftbildes typisch. Sie ist der evolutive Vorläufer der Kleinbuchstaben deutscher Schriftarten und der lateinischen Schrift. Zusammen mit den althochdeutschen Randbemerkungen des 9. und 10. Jh., die ein Beleg für die intensive Auseinandersetzung mit den Inhalten sind, macht diese Art der handschriftlichen Gestaltung das Lorscher Arzneibuch zu einem wertvollen Dokument für die germanische Sprachwissenschaft. Auch diese Medizinwissenschaft-übergreifende Bedeutung unterstreicht die Einmaligkeit des Werkes.

Hinzu kommt das im Buch befindliche (Teil-) Bücherverzeichnis einer kaiserlichen Bibliothek des Frühmittelalters, die Leo von Vercelli als Lehrer und Vertrauter KaIser OtTos II. zusammengestellt hatte. Als einzig bekanntes Dokument dieser Art bietet es auf diese Weise eine Vielzahl von Ansatzpunkten zur Rekonstruktion der Entstehung und Veränderung des Lorscher Arzneibuches.

\section{Besonderer Stellenwert}

Eine der wichtigsten, den hohen weltweit bedeutsamen Stellenwert kennzeichnende Botschaft dürfte jedoch die Verknüpfung einer christlich motivierten Selbstverpflichtung zum Helfen mit der säkularen Wissenschaft sein. Es handelt sich um eine dem Mönchtum geschuldete neue Denk- und Handlungsweise in Bezug auf die Heilkunst und das Christentum.

\section{Literatur \\ Fuchs, L. 1543: Das Kräuterbuch von 1543. - Reprint Köln 2001.}

\section{Weiterführende Internetseiten \\ Leseprobe unter: http://books.google.de/books?id=Jr2NQ CevkUUC\&pg=PA 275\&lpg=PA275\&dq=Lorscher+Arznei buch+Johanniskraut\&source $=$ bl\&ots=91 nnnBKJib\&sig $=\mathrm{V}$ ULJRc_tUZkgNtrgY1ZZCmJ8- mg\&hl=de\&sa =X\&ei=PszFUf- dLMf_4QSOoYHAAg\&ved=0CE8Q6AEwBg\#v=onepage $\& \mathrm{q}=$ Lorscher\%20Arzneibuch\%20Johanniskraut\&f=false Volldigitalisiert unter: www.staatsbibliothek-bamberg.de http://www.unesco.de/80006/html http://www.klostermedizin.de/index. php?art=95\&markierung=bei \\ Pressemitteilung der Staatsbibliothek Bamberg von Prof. Dr. Werner Taegert vom 19.6.2013 \\ http://www.kloster-lorsch.de/kloster/arznei.html}

Abb. 6: Das Lorscher Arzneibuch enthält im Gegensatz zu vielen alten Kräuterbüchern keine Illustrationen. Hier ein Salbei, eine klassische Heilpflanze, aus dem Kräuterbuch des Leonhart Fuchs von 1543. 